\title{
Failure of Postnatal Adaptation of the Pulmonary Circulation after Chronic Intrauterine Pulmonary Hypertension in Fetal Lambs
}

Steven H. Abman, Paul F. Shanley, and Frank J. Accurso

Departments of Pediatrics and Pathology and the Division of Perinatal Medicine, the University of Colorado School of Medicine, Denver, Colorado 80262

\begin{abstract}
To determine the effects of chronic intrauterine pulmonary hypertension on the perinatal pulmonary circulation, we induced chronic elevations of pulmonary artery pressure in 24 late-gestation fetal lambs by maintaining partial compression of the ductus arteriosus with an inflatable vascular occluder. Pulmonary artery pressure was increased from $44 \pm 1$ to $62 \pm 3$ mmHg for 3-14 d. Although left pulmonary artery blood flow initially increased during acute partial ductus compression, the increase in flow was not sustained during chronic ductus compression despite persistent elevations of pulmonary artery pressure $(P<0.01)$. Chronic hypertension decreased the slope of the pressure-flow relationship from $3.4 \pm 0.3$ (initial) to $0.9 \pm 0.1 \mathrm{ml} / \mathrm{min}$ per $\mathrm{mmHg}$, and blunted the fetal pulmonary vascular response to small increases in $\mathrm{PO}_{2}(P<0.0001)$. Pulmonary hypertension for $>\mathbf{8 d}$ increased the wall thickness of small pulmonary arteries $(P<0.001)$. Compared with controls, hypertensive animals had higher pulmonary artery pressure, lower pulmonary blood flow, and predominant right-to-left ductus shunting after cesarean-section delivery $(P<0.0001)$. We conclude that chronic pulmonary hypertension in utero, in the absence of hypoxemia or sustained increases in blood flow, causes abnormal fetal pulmonary vasoreactivity, structural remodeling, and the failure to achieve the normal decline in pulmonary resistance at birth.
\end{abstract}

\section{Introduction}

Within minutes of birth, the normal fetus undergoes a dramatic transition from its dependence on the placenta for gas exchange to the establishment of adequate ventilation and perfusion of the lung. These early cardiopulmonary events include a 6-10-fold rise in pulmonary blood flow and a decline in pulmonary vascular resistance, increased systemic vascular resistance with the loss of the low resistance umbilical circuit and increased neurohumoral stimuli, functional closure of the foramen ovale, and closure of the ductus arteriosus (1-4). Although mechanisms contributing to the normal transition of the pulmonary circulation are not completely understood, increased oxygen tension, ventilation, the development of an air-liquid interface, and perhaps the release of vasoactive me-

Address reprint requests to Dr. Steven H. Abman, Box C-220, Department of Pediatrics, University of Colorado School of Medicine, 4200 E. Ninth Avenue, Denver, CO 80262.

Received for publication 19 July 1988 and in revised form 31 January 1989.

J. Clin. Invest.

(C) The American Society for Clinical Investigation, Inc.

$0021-9738 / 89 / 06 / 1849 / 10 \quad \$ 2.00$

Volume 83, June 1989, 1849-1858 diators appear to play important roles in this adaptation $(1,2$, $4,5)$.

Some infants develop cardiopulmonary distress in the immediate newborn period, and are unable to successfully achieve or sustain the postnatal decline in pulmonary vascular resistance $(6,7)$. This syndrome, known as persistent pulmonary hypertension of the newborn (PPHN), ${ }^{1}$ is associated with a wide variety of neonatal disorders, including asphyxia, sepsis, meconium aspiration, diaphragmatic hernia, congenital heart disease, hyaline membrane disease, and others, or can be idiopathic ("persistent fetal circulation") (8). Despite the diversity of associated clinical conditions, all of these disorders share a common pathophysiology. That is, the failure of postnatal adaptation of the pulmonary circulation at birth is characterized by elevated pulmonary artery pressure and abnormal pulmonary vascular reactivity, both of which lead to right-to-left shunting across the ductus arteriosus or foramen ovale, contributing to severe hypoxemia and the high morbidity and mortality of PPHN (6-9).

Based on several clinical and experimental studies, intrauterine events appear to be related to the development of PPHN (9). First, the timing of onset of the disease is generally within hours of birth. In addition, infants dying within the first few days of life with PPHN frequently have striking pulmonary vascular structural changes, including smooth muscle and adventitial thickening, suggestive of a chronic intrauterine process (10). Finally, data from animal studies suggest that chronic fetal hypertension or hypoxia may cause structural lesions similar to those found in humans with PPHN (11-17). However, recent attempts to demonstrate physiologic or histologic abnormalities after chronic intrauterine hypoxia or indomethacin treatment have not supported this hypothesis, and mechanisms contributing to the initiation and progression of the pulmonary vascular abnormalities of PPHN are unknown (18-20). In addition, no study has actually linked intrauterine events with altered perinatal pulmonary vasoreactivity, the inability to achieve or sustain a drop in pulmonary vascular resistance at birth, and structural vascular changes.

Therefore, to determine if intrauterine events can alter fetal pulmonary vascular reactivity and structure, and adaptation of the pulmonary circulation to postnatal conditions during the immediate newborn period, we performed a series of experiments examining the chronic effects of partial compression of the ductus arteriosus in fetal sheep.

\section{Methods}

Animal preparation

24 mixed breed (Columbia-Rambouillet) pregnant ewes between 119 and $127 \mathrm{~d}$ gestation (term $=147 \mathrm{~d}$ ) were fasted for at least $24 \mathrm{~h}$ before surgery. Ewes were sedated under intravenous pentobarbital and anes-

1. Abbreviations used in this paper: $\mathrm{ED}$, external diameter; $\mathrm{LV}+\mathrm{S}$, left ventricle plus septum; PPHN, persistent pulmonary hypertension of the newborn; RV, right ventricle; WT, wall thickness. 
thetized with $1 \%$ tetracaine hydrochloride by lumbar injection ( $3 \mathrm{mg}$ ). Under sterile conditions, the fetal left forelimb was delivered through a uterine incision. A left fetal thoracotomy exposed the heart and great vessels. Polyvinyl catheters were inserted into the left and main pulmonary arteries, and left atrium by direct puncture after the placement of purse string sutures, as previously described $(21,22)$. Catheters were guided into position with a no. 14 or no. 16 gauge intravenous placement unit (Angiocath; Travenol, Deerfield, IL) modified to minimize blood loss. The catheters were secured by tightening the purse string suture as the introducer was withdrawn. The left pulmonary artery catheter was inserted at the bifurcation of the main pulmonary artery and ductus arteriosus. The main pulmonary artery catheter was inserted between the ductus arteriosus and pulmonic valve. The left atrial catheter was placed in a medial portion of the left atrial appendage. An inflatable vascular occluder was placed loosely around the ductus arteriosus after gentle dissection of adherent connective tissue with cotton-tipped swabs. Occluder sizes were selected to allow for minimal compression of the ductus arteriosus until the time of study. A cuff-type electromagnetic flow transducer (Micron Instruments, Los Angeles, CA) was placed around the left pulmonary artery to measure blood flow to the left lung. Sizes varied between 3.0 and $4.5 \mathrm{~mm}$, and were selected to make adequate contact with the vessel with minimal compression of the left pulmonary artery. Flow transducers were previously calibrated with saline infusions through carotid arteries of adult sheep in situ. Additional catheters were placed in the fetal abdominal aorta and inferior vena cava via the pedal artery and vein. A catheter was placed in the amniotic cavity to measure pressure. After closure of the hysterotomy and return of the uterus to the ewe's abdominal cavity, the occluder, flow transducer cables, and catheters were exteriorized via subcutaneous tunnels to an external flank pouch. The ewes recovered rapidly from surgery, and were generally standing in their pens within $6 \mathrm{~h}$. Food and water was allowed ad libitum. Catheters were maintained by daily infusions of 2-3 $\mathrm{ml}$ of heparinized saline $(35 \mathrm{U} / \mathrm{ml})$. Catheter positions were verified at autopsy. Studies were performed after a minimum postoperative recovery period of $72 \mathrm{~h}$.

The same surgical protocol was followed in the preparation of 18 chronically instrumented fetal animals for physiologic studies in control animals, except for the inflatable ductus occluder, which was placed in three control animals.

\section{Physiologic measurements}

Flow transducer cables were attached to an internally calibrated flowmeter (Micron Instruments), providing continuous measurements of left pulmonary blood flow. Calculations for the absolute values of flow were determined from phasic blood flow signals, with zero blood flow defined as the stationary portion of the flow signal occurring during late diastole following the negative component of flow (22-24). Previous studies in which blood flow was measured at the time of killing verified the accuracy of this method within $20 \mathrm{ml} / \mathrm{min}$ (21). Pulmonary artery, aortic, amniotic cavity, and left atrial pressures were connected to a pressure transducer (model P23 ID; Gould-Statham Inc., Cleveland, $\mathrm{OH}$ ). Pressures were referenced to the amniotic pressure. Calibrations of pressure transducers were performed with a mercury column manometer. Heart rate was̀ continuously recorded from cardiotachometer or determined from phasic pulmonary blood flow tracings. Measurements were continuously recorded on a chart recorder (Gould-Statham, Ine.). Pulmonary vascular resistance was calculated as the difference between mean pulmonary artery and left atrial pressures divided by flow. Left atrial pressure was measured in seven animals. Because left atrial pressure did not change with partial compression of the ductus arteriosus, left atrial pressure was estimated at 2 $\mathrm{mmHg}$ for the other studies.

Oxygen saturation was measured from main pulmonary artery samples by a hemoximeter (model OSM-2; Radiometer, Copenhagen); blood gas tensions and $\mathrm{pH}$ were measured at $39.5^{\circ} \mathrm{C}$ by an analyzer (model BM53 Mark 2; Radiometer).

\section{Experimental design}

Four experimental protocols are included in this study: 1, the fetal hemodynamic effects of chronic partial compression of the ductus arteriosus; 2 , the effects of chronic partial ductus compression on fetal pulmonary vasoreactivity; 3 , structural effects of chronic pulmonary hypertension on small pulmonary arteries; and 4 , the effects of chronic intrauterine pulmonary hypertension on postnatal adaptation, as determined by the hemodynamic response after cesarean-section delivery. Chronic ductus compression was successfully maintained in 24 animals, starting at a mean gestational age of $128 \mathrm{~d}$ (range: 124-133 d; protocol 1). The study was not completed in seven animals because of the late onset of hypoxemia, intrauterine demise, or premature delivery. Fetal pulmonary vasoreactivity (protocol 2 ) was assessed by determinations of the pressure-flow relationship before and after chronic ductus compression ( $n=12$ animals), and the vasodilator response to small increases in fetal $\mathrm{PO}_{2}$ ( $n=7$ study and 12 control animals). 12 study and 12 control animals were killed after chronic partial ductus compression for morphometric analysis (protocol 3). Cesarean-section deliveries (protocol 4) were performed in six control and five study animals.

Protocol 1. Hemodynamic effects of chronic partial compression of the ductus arteriosus ( $n=24$ animals). After at least $3 \mathrm{~d}$ recovery from the acute stresses of surgery, the occluder surrounding the ductus arteriosus was partially inflated to maintain chronic elevations of pulmonary artery pressure. Initial hemodynamic measurements including mean pulmonary artery pressure, mean aorta pressure, left pulmonary artery blood flow, heart rate, $\mathrm{O}_{2}$ saturation, and hematocrit were recorded before the initial inflation of the ductus occluder, and after chronic compression (mean duration, $8 \mathrm{~d}$; range: $3-14 \mathrm{~d}$ ). To achieve the highest degree of pulmonary hypertension without causing hypoxemia, oxygen saturation, hematocrit, heart rate, and mean main pulmonary and aorta pressures were measured daily during chronic ductus compression. The degree of partial ductus compression was based on observations that moderate compression frequently lead to hypoxemia, especially over time. During the initial period of chronic ductus compression, the degree of inflation of the occluder often had to be cautiously adjusted on a daily basis, with the degree of occlusion generally increasing over time. If oxygen saturations in main pulmonary artery blood specimens were $<45 \%$, the degree of compression was diminished or occlusion completely released until an improvement in oxygen saturation was noted on subsequent days. Oxygen saturations were measured immediately after the acute release of the ductus occluder, as well as on at least a daily basis. Animals with oxygen saturations that persisted $<45 \%$ were removed from the study.

Protocol 2. Effects of chronic partial ductus compression on fetal pulmonary vascular reactivity. The effects of chronic partial ductus compression on fetal pulmonary vascular reactivity was assessed by determining $(a)$ the changes in the pressure-flow relationship of the fetal pulmonary circulation ( $n=12$ study animals), and $(b)$ the hemodynamic response to small increases in fetal oxygen tension after chronic pulmonary hypertension ( $n=7$ study and 12 control animals).

Abrupt partial inflation of the ductus occluder increases pulmonary artery pressure, and mean and phasic blood flow to the left lung, which return to baseline values after release of the occluder (Fig. 1). Using this capability to control pulmonary artery pressure while measuring changes in flow, we assess pressure-flow relationships of the fetal pulmonary circulation by determining changes in mean flow in response to small, rapid incremental compressions of the ductus (25). Progressive partial inflations of the ductus occluder increased pulmonary artery pressure in stepwise fashion, without changing left atrial pressure. Mean blood flow followed the incremental changes in pressure. The entire procedure was performed in $<1 \mathrm{~min}$. Linear plots of mean pulmonary artery pressure and flow from at least four time points describe the pressure-flow relationship of the fetal pulmonary circulation. Vascular conductance is determined from the slope of the pressure-flow relationship, and expressed as milliliters/minutes per millimeters of mercury. The reproducibility of this technique was de- 
Q Mean 200 . (mi/min) 100-

termined from repeated measurements at least 15 min apart in eight animals, with slopes varying $<20 \%$ for all studies (25).

The fetal pulmonary vascular response to increases in $\mathrm{PO}_{2}$ was studied before and during the administration of $100 \%$ oxygen to the ewe. After recording baseline hemodynamic and arterial blood gas tension measurements for at least 30 min, $100 \%$ oxygen was administered to the ewe for $60 \mathrm{~min}$ through a plastic bag tied snugly around the ewe's head, as previously described $(26,27)$. Flow rates varied between 45 and 60 liters $/ \mathrm{min}$. Comparisons were made between 7 animals treated with chronic ductus compression (mean gestational age, $133 \pm 2$ d) and a control group of 12 age-matched fetal lambs (134 \pm 1 d) that had undergone fetal surgery as described for the hypertensive group, but without chronic ductus compression. Two of these control animals had inflatable vascular occluders placed around the ductus arteriosus but did not undergo chronic compression.

Protocol 3. Structural effects of chronic intrauterine pulmonary hypertension on small pulmonary arteries. Autopsies were performed immediately after killing with the euthanasia compound T-61 (Taylor Pharmaceutical, Sparks, MD). Fetal weight was recorded, and catheter, occluder, and flow transducer positions were confirmed after thoracotomy. The trachea and main pulmonary artery were isolated, cannulated with large bore polyethylene tubing, and simultaneously perfused with $10 \%$ buffered formalin. Perfusion pressure was $30 \mathrm{~cm} \mathrm{H}_{2} \mathrm{O}$ for airway fixation; the vascular perfusion pressure was identical to the mean main pulmonary artery pressure measured immediately before killing. During perfusion, the ductus arteriosus was closed by full inflation of the occluder, or ligated (in control animals). After fixation, thin $(\sim 0.5 \mathrm{~cm})$ longitudinal sections were carefully removed from the right and left lower lobes of the lungs and stored in formalin. After dehydration through graded alcohols, and embedding in paraffin, $5-\mu \mathrm{m}$ sections were stained with hematoxylin and eosin, Masson trichrone, and Verhoff-Van Gieson stains.

In each microscopic specimen, a minimum of 12 small pulmonary arteries landmarked by their association with small terminal bronchioles were measured. These bronchioles were defined as the smallest airway leading to an acinus that had simple columnar or cuboidal epithelium on both sides of the airway. These vessels were selected for measurements to ensure consistency for comparisons between the study groups (outlined below). Each artery was measured with the interactive digital analyzer system (Carl Zeiss, Inc., Thornwood, NY); the data were passed to an IBM PC/XT computer via an asynchronous communications adapter. External diameter (ED) and wall thickness (WT) were measured directly; lumen diameter (ED $-2 \times$ WT) and percent wall thickness $(2 \times$ WT $\times 100 / E D)$ were calculated from these values. Measurements were made in blinded fashion; interobserver variability was assessed and found to be minimal by comparing measurements from similar slides.

To assess the development of right ventricular hypertrophy, the ratio of the weights of the right ventricle (RV) to left ventricle plus septum $(L V+S)$ was determined (28). At autopsy, the free wall of the right ventricle was removed from the fetal heart and weighed separately from the $L V+S$. The $L V$ free wall was not weighed separately from the septum.
Figure 1. Physiologic tracing demonstrates the rapid rise in pulmonary artery pressure (top) and left pulmonary artery blood flow during partial inflation of the occluder surrounding the ductus arteriosus in a late-gestation animal.

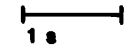

Comparisons were made between three groups of animals: group I, control (no ductus compression, $n=12$ ); group II, brief hypertension (exposed to 3-6 d of compression, $n=6$ ); and group III, prolonged hypertension (9-14 d of compression, $n=6$ ).

Protocol 4. Effects of chronic intrauterine pulmonary hypertension on the hemodynamic response to cesarean-section deliveries. Cesarean-section deliveries were performed under intravenous pentobarbitol anesthesia to the ewe. The uterus was partially delivered through an abdominal incision, and the flow transducer cables and catheters were carefully freed from the maternal flank. The ductus occluder was released at least $1 \mathrm{~h}$ before delivery. Baseline hemodynamic, hematocrit, $\mathrm{O}_{2}$ saturation, and arterial blood gas tension, and $\mathrm{pH}$ measurements were obtained for at least $30 \mathrm{~min}$ before delivery. After the injection of pancuronium bromide ( $1 \mathrm{mg}$, inferior vena cava) to the fetus, a hysterotomy was performed, and the fetus was rapidly intubated with a cuffed 5.0 endotracheal tube. The umbilical cord was cut, and the fetus was extracted from the uterus while being hand-ventilated with $100 \%$ oxygen. The animal was dried and warmed, placed on a heating pad, and ventilated with a time-cycled, pressure-limited mechanical ventilator. Initial settings were as follows: peak inspiratory pressure, 24-28 $\mathrm{cm} \mathrm{H}_{2} \mathrm{O}$; peak end-expiratory pressure, $4 \mathrm{~cm} \mathrm{H} \mathrm{H}_{2} \mathrm{O}$; rate, $30 \mathrm{bpm}$; inspiratory to expiratory ratio, 1:3; and fraction of inspired oxygen concentration, 1.00. Lambs were kept on a heating pad throughout the study, and temperature was continuously monitored with a rectal probe. Intravenous fluids ( $5 \%$ dextrose in 0.2 normal saline) were infused at $12 \mathrm{ml} / \mathrm{h}$. The concentration of inspired oxygen was intermittently monitored with an oxygen analyzer. Serial measurements were recorded at 10-min intervals for at least $2 \mathrm{~h}$ after delivery. During the study period, ventilator settings were changed to maintain arterial $\mathrm{PO}_{2}$ $<50$ Torr (in descending aorta samples) and $\mathrm{pH}>7.20$. Inspired oxygen concentrations were adjusted to maintain $\mathrm{PO}_{2}>80$ Torr. In three animals, an arterial line was placed in the right forelimb for preductal arterial blood gas sampling.

Comparisons were made with six control animals that had undergone fetal surgery between 120 and 125 d gestation, but were not treated with chronic ductus compression. At thoracotomy, each animal had catheters and an electromagnetic flow transducer placed on the left pulmonary artery, as described above. In addition, two control animals had an inflatable occluder placed around the ductus, but were not treated with chronic compression.

\section{Data analysis}

Statistical analysis of the data was performed by two-way analysis of variance for repeated measures with a commercially available statistics package (SAS Institute, Cary, NC) on a VAX minicomputer (29). Slopes of the pressure-flow relationships (protocol 2) and cardiac weights (protocol 3) were analyzed by $t$ test. $P$ values $<0.05$ were considered significant.

\section{Results}

Hemodynamic effects of chronic partial compression of the ductus arteriosus (protocol 1). Serial hemodynamic, hematocrit, and $\mathrm{O}_{2}$ saturation measurements before and after chronic 
partial compression of the ductus arteriosus are shown in Table I. Mean pulmonary artery pressure was increased from $44 \pm 1$ (mean \pm SEM) to $62 \pm 3 \mathrm{mmHg}$ over $8 \pm 1 \mathrm{~d}$ (range: $3-14$ ). The increases in mean aorta pressure (from $40 \pm 1$ to $45 \pm 1$ $\mathrm{mmHg}$ ) and hematocrit ( $37 \pm 1$ to $39 \pm 1 \%$ ) were small, but statistically significant. Left atrial pressure, as determined from seven animals, did not change from initial values $(2 \pm 1$ $\mathrm{mmHg}) . \mathrm{O}_{2}$ saturation from main pulmonary artery blood samples decreased slightly, from $56 \pm 1$ to $50 \pm 1 \%$, but remained above ranges generally considered hypoxic for the fetus (30). Although partial ductus compression initially leads to a rise in blood flow and a decline in resistance, these responses were not sustained during chronic ductus compression (25). With the acute partial compression of the ductus arteriosus, there was a rapid rise in blood flow from $64 \pm 2$ to $135 \pm 8$ $\mathrm{ml} / \mathrm{min}$ at $30 \mathrm{~min}$, as mean pulmonary artery pressure was increased from $44 \pm 1$ to $63 \pm 5 \mathrm{mmHg}$. As shown in Table I, after $8 \mathrm{~d}$ of partial ductus compression, blood flow was not significantly different from baseline values prior to compression, despite the increase in pulmonary artery pressure to $62 \pm 3 \mathrm{mmHg}$. Thus, not only was the initial decline in pulmonary vascular resistance not sustained, resistance actually increased from $0.72 \pm 0.05$ (baseline) to $1.08 \pm 0.08 \mathrm{mmHg} / \mathrm{ml}$ per min after chronic compression $(P<0.001)$.

Effects of chronic pulmonary hypertension on fetal pulmonary vasoreactivity (protocol 2). To further assess the change in pulmonary vascular resistance after chronic partial ductus compression, we determined pressure-flow relationships of the fetal pulmonary circulation before and after chronic hypertension. As illustrated for a single animal (Fig. 2, left) and shown for 12 hypertensive animals (right), the slope of the pressureflow relationship decreased from $3.4 \pm 0.3$ to $0.9 \pm 0.1 \mathrm{ml} / \mathrm{min}$ per $\mathrm{mmHg}$ after partial compression of the ductus arteriosus
$(P<0.001)$. This suggests that chronic pulmonary hypertension in utero decreases pulmonary vascular conductance.

The effects of chronic pulmonary hypertension on the fetal hemodynamic response to small increases in fetal $\mathrm{Po}_{2}$ is shown in Fig. 3. In the control animals, small increases in fetal $\mathrm{PO}_{2}$ during the administration of $100 \% \mathrm{O}_{2}$ to the ewe (as measured from main pulmonary artery samples; Fig. 3, top) increased left pulmonary blood flow from $66 \pm 7$ to $165 \pm 23 \mathrm{ml} / \mathrm{min}$ at 60 min $(P<0.0001)$. In contrast, in the seven animals treated with chronic partial ductus compression, left pulmonary artery blood flow did not increase, despite an equivalent rise in fetal $\mathrm{PO}_{2}$. Heart rate, mean aorta pressure, $\mathrm{pH}$ and $\mathrm{PCO}_{2}$ did not significantly change from baseline values during the study period.

Structural effects of chronic intrauterine pulmonary hypertension on small pulmonary arteries (protocol 3). Histologic examination of small fetal pulmonary arteries after at least $9 \mathrm{~d}$ of ductus compression revealed striking differences in comparison with normal late-gestation animals (Fig. 4). As illustrated, lumina were more narrowed and the smooth muscle appeared thicker. Morphometric analysis of small pulmonary arteries associated with terminal bronchioles was used to quantitate differences between control and hypertensive animals, as well as to determine the duration of hypertension necessary to develop these structural changes. As shown in Table II, the external diameters of arteries associated with terminal bronchioles were not different between groups. However, the wall thickness was much greater after 9-14 d of hypertension (10.8 $\mathrm{d}$, mean) than control or brief hypertension (3.8 d, mean; range: $3-6 \mathrm{~d})$ animals $(P<0.001)$. Similarly, the percent wall thickness was greater, and the lumen diameter smaller after prolonged, but not brief, hypertension. In addition, the ratio of the weights of the right ventricle to the left ventricle and sep-

Table I. Changes in Hemodynamic, Hematocrit, and $\mathrm{O}_{2}$ Saturation Measurements before and after Chronic Partial Compression of the Ductus Arteriosus

\begin{tabular}{|c|c|c|c|}
\hline Parameter & Initial & Final & $P^{*}$ \\
\hline \multicolumn{4}{|l|}{ Gestational age $(d)$} \\
\hline Control & $127 \pm 2]$ & \multirow{2}{*}{$s$} & 一 \\
\hline Hypertensive & $128 \pm 1]^{\mathrm{NS}}$ & & - \\
\hline \multicolumn{4}{|c|}{ Mean pulmonary artery pressure $(\mathrm{mm} \mathrm{Hg})$} \\
\hline Control & $41 \pm 1] \mathrm{NS}$ & \multirow{2}{*}{]$<0.001$} & $<0.05$ \\
\hline Hypertensive & $44 \pm 1]^{\text {NS }}$ & & $<0.001$ \\
\hline \multicolumn{4}{|l|}{ Mean aorta pressure } \\
\hline Control & $39 \pm 1] \mathrm{NS}$ & \multirow{2}{*}{ NS } & $<0.01$ \\
\hline Hypertensive & $40 \pm 1]^{N S}$ & & $<0.01$ \\
\hline \multicolumn{4}{|l|}{ LPA Blood Flow ( $\mathrm{ml} / \mathrm{min})$} \\
\hline Control & $66 \pm 4] \mathrm{NS}$ & \multirow{2}{*}{]$<0.05$} & $<0.02$ \\
\hline Hypertensive & $62 \pm 4]^{N S}$ & & NS \\
\hline \multicolumn{4}{|l|}{ PVR $(m m H g / m l$ per min $)$} \\
\hline Control & $0.69 \pm 0.05]$ & $\left.\begin{array}{l}6 \\
8\end{array}\right]<0.01$ & NS \\
\hline Hypertensive & $0.72 \pm 0.05]^{\mathrm{NS}}$ & $1.08 \pm 0.08]<0.01$ & $<0.001$ \\
\hline \multicolumn{4}{|l|}{$\mathrm{O}_{2}$ Saturation (\%) } \\
\hline Control & & \multirow{2}{*}{ NS } & NS \\
\hline Hypertensive & $56 \pm 1] \mathrm{NS}$ & & $<0.01$ \\
\hline
\end{tabular}

Data are expressed as mean \pm SEM. Comparisons between control and hypertensive animals were by nonpaired $t$ test. Abbreviations: LPA, left pulmonary artery; PVR, pulmonary vascular resistance. The $\mathrm{O}_{2}$ saturation was measured from main pulmonary artery samples. ${ }^{*} P$ values in this column compare initial versus final values by paired $t$ test. 

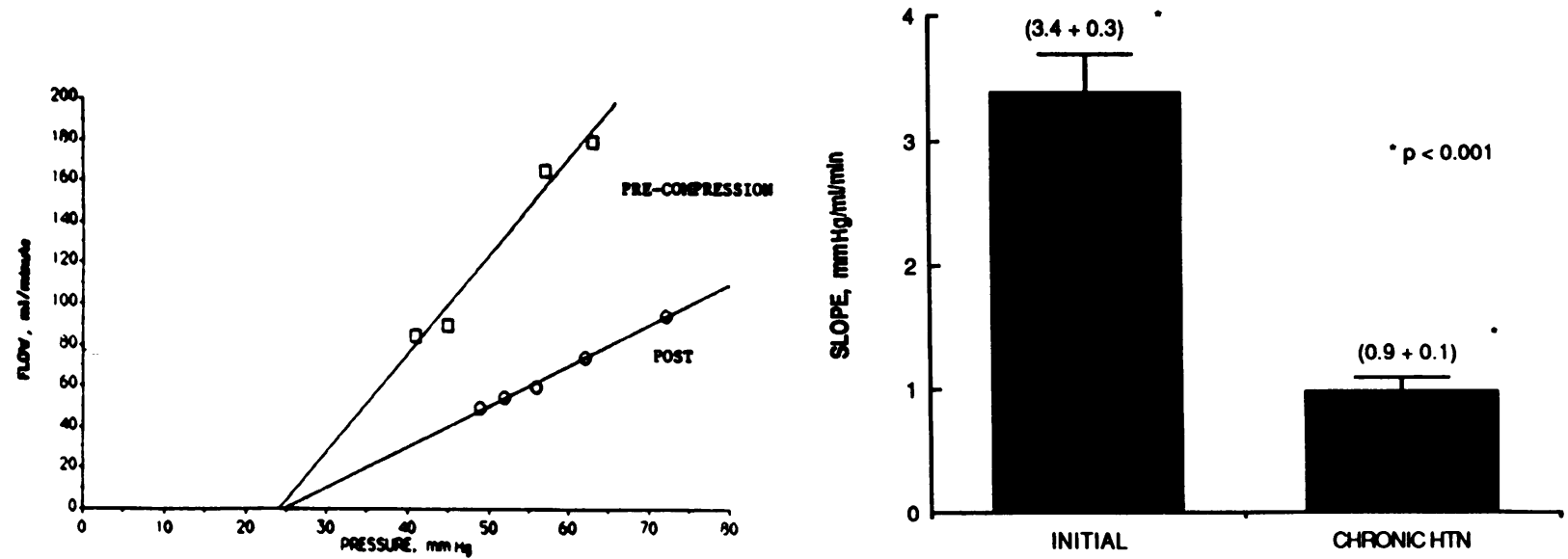

Figure 2. Effects of chronic partial compression of the ductus arteriosus on the slope of the pressure-flow relationship (vascular conductance). (Left) The change in slope of the pressure-flow relationship before and after $5 \mathrm{~d}$ of hypertension in a late-gestation fetal lamb. (Right) The change in slope before and after chronic ductus compression for the entire study group.

tum was increased after chronic ductus compression, suggesting the development of right ventricular hypertrophy (Table III). Although the right ventricular weight was greater after hypertension, the weight of the left ventricle plus septum was similar between groups. Because fetal weights were similar between groups, these differences persist when expressed on a per kilogram basis. The ratio of right ventricular to left ventricular plus septum was greater after prolonged ductus compression than brief hypertension $(0.63 \pm 0.02$ vs. $0.55 \pm 0.02, P<0.01)$.

Effects of chronic intrauterine pulmonary hypertension on the hemodynamic response after cesarean-section delivery (protocol 4). To determine the effects of intrauterine pulmo-
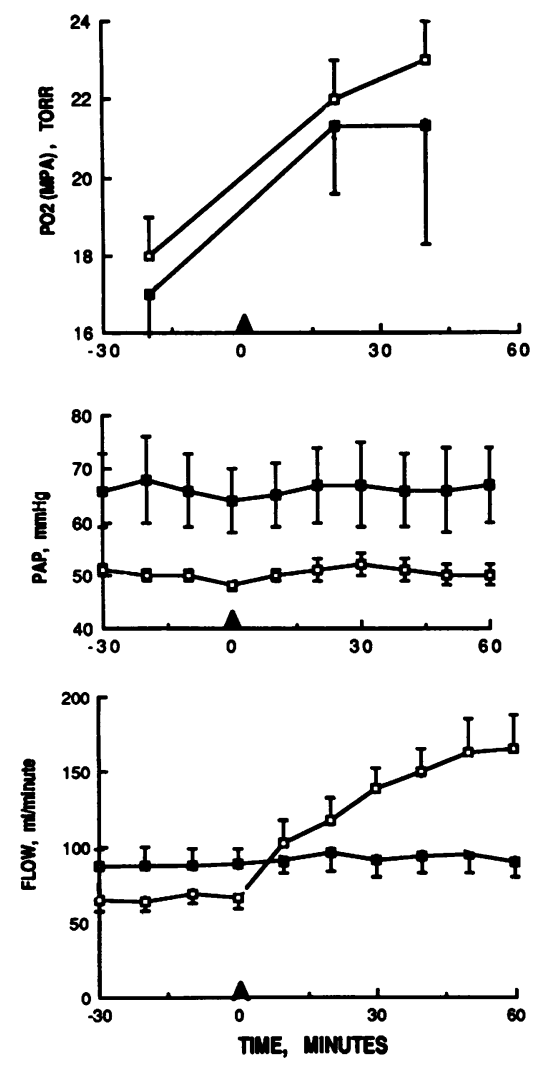

Figure 3. Effects of chronic partial ductus compression on the hemodynamic response to increases in fetal $\mathrm{PO}_{2}$. (Top) Arterial $\mathrm{PO}_{2}$ from main pulmonary artery samples in control ( $\square$ ) and study animals (ם). (Middle and bottom) Mean pulmonary artery pressure and pulmonary blood flow, respectively. The rise in pulmonary blood flow during increases of fetal $\mathrm{PO}_{2}$ are significantly less in hypertensive animals throughout the study period $(P<0.0001$, control versus hypertensive animals by repeated measures ANOVA). nary hypertension on adaptation to postnatal conditions, we delivered five animals by cesarean-section after $11 \pm 2 \mathrm{~d}$ of partial ductus compression (range: 9-12 d). Responses to cesarean-section delivery were compared with six control animals. Gestational ages were $143 \pm 2$ and $141 \pm 1 \mathrm{~d}$ for control and hypertensive animals, respectively. All animals had undergone surgery between 120 and $125 \mathrm{~d}$, preceding cesarean-section delivery by a mean duration of $16 \mathrm{~d}$ (control group) and $17 \mathrm{~d}$ (hypertensive animals). The hypertensive animals received 9-12 d of partial ductus compression (mean, $11 \mathrm{~d}$ ). Initial hematocrit ( $43 \pm 1 \%$ [control] versus $40 \pm 3 \%$ [hypertension]), blood gas tension, and $\mathrm{pH}$ were not different at the time of delivery (Table IV).

Fig. 5 displays the hemodynamic responses of mean pulmonary artery pressure, mean aorta pressure, and left pulmonary artery blood flow before and after delivery. As shown, the initial pulmonary artery pressure was significantly greater than control animals during the baseline period ( $62 \pm 5$ [hypertensive] vs. $56 \pm 2 \mathrm{mmHg}$ [control] $P<0.01$ ). By $2 \mathrm{~h}$, mean pulmonary artery pressure had decreased to $39 \pm 4 \mathrm{mmHg}$ in control animals, representing a $30 \%$ decline. In contrast, the mean pulmonary pressure did not significantly decrease after delivery in the hypertensive group, and was $59 \pm 2 \mathrm{mmHg}$ at $2 \mathrm{~h}$ after delivery, despite ventilation with $100 \% \mathrm{O}_{2}$. Mean aortic pressure (Fig. 5, middle) was not different between the groups during the baseline period, and increased significantly with birth in both groups. However, mean aorta pressure was significantly greater in the hypertensive animals during the postdelivery period $(P<0.01)$. Left pulmonary artery blood flow in the controls (Fig. 5, bottom) increased from $52 \pm 6 \mathrm{ml} / \mathrm{min} \mathrm{im}$ mediately before delivery to $297 \pm 34 \mathrm{ml} / \mathrm{min}$ at $10 \mathrm{~min}$, and remained elevated at $2 \mathrm{~h}(289 \pm 37 \mathrm{ml} / \mathrm{min})$, representing a 5.7-fold increase. Although pulmonary blood flow during the baseline period was not different between the groups $(59 \pm 6$ [hypertensive] vs. $52 \pm 6 \mathrm{ml} / \mathrm{min}$ [control]), flow was significantly less in the hypertensive animals than in the control group throughout the postdelivery study period $(P<0.0001)$. Left pulmonary artery blood flow in the hypertensive animals increased 2.4-fold, to $139 \pm 33 \mathrm{ml} / \mathrm{min} 10 \mathrm{~min}$ after delivery, and $120 \pm 38 \mathrm{ml} / \mathrm{min}$ at $2 \mathrm{~h}$.

In addition to the marked elevation of pulmonary artery and aorta pressures after delivery of animals exposed to 

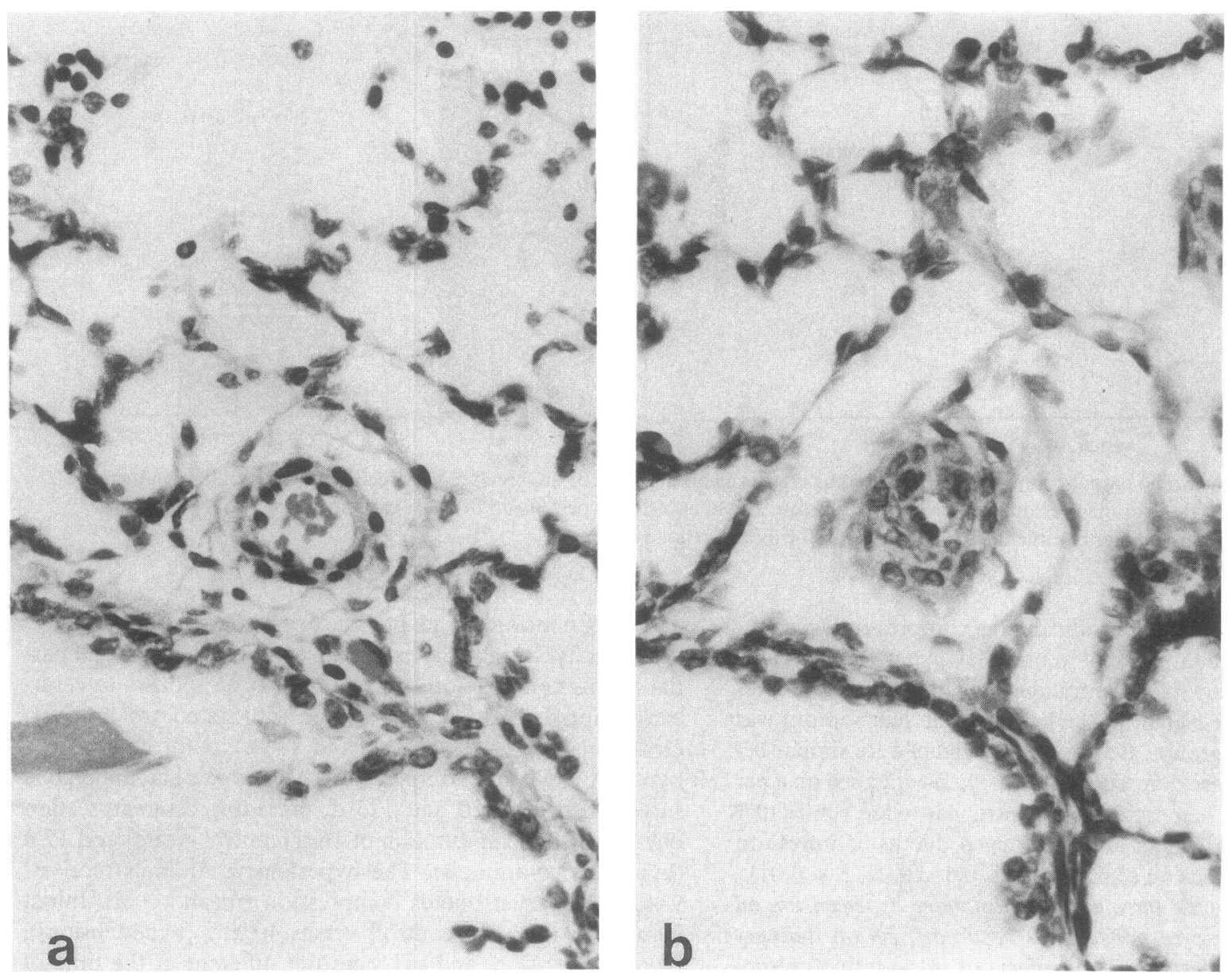

Figure 4. Effects of pulmonary hypertension secondary to partial compression of the ductus arteriosus on the morphology of small pulmonary arteries. Comparable vessels associated with terminal bronchioles are shown in late gestation animals from $(a)$ a control animal and $(b)$ after 12 d of partial ductus compression. Note the marked increase in wall thickness and narrowing of lumen diameter in the hypertensive vessel. Hematoxylin-eosin, $\times 500$.

chronic hypertension, the ratio of mean pulmonary artery to aorta pressure was also greater than control values $(P<0.001$; Fig. 6). In control animals, mean pulmonary artery pressure was $67 \pm 6 \%$ of systemic pressure at $2 \mathrm{~h}$. In contrast, mean pulmonary artery pressure was near systemic levels in the hypertensive group $(0.97 \pm 4 \%)$. In addition, pulmonary resistance was markedly increased in the hypertensive animals

Table II. Morphometric Analysis of Small Pulmonary Arteries Associated with Terminal Bronchioles in Control and Hypertensive Animals

\begin{tabular}{lcccc}
\hline & $\begin{array}{c}\text { External } \\
\text { diameter }\end{array}$ & Wall thickness & $\begin{array}{c}\text { Lumen } \\
\text { diameter }\end{array}$ & Wall thickness \\
\hline & & $\mu M$ & & $\%$ \\
Control $(n=12)$ & $21.2 \pm 0.3$ & $5.6 \pm 0.1$ & $10.0 \pm 0.6$ & $53.3 \pm 2.8$ \\
Brief htn $(n=6)$ & $22.0 \pm 0.6$ & $4.9 \pm 0.2$ & $11.4 \pm 0.7$ & $47.3 \pm 3.0$ \\
Long htn $(n=6)$ & $20.2 \pm 0.5$ & $7.8 \pm 0.3^{*}$ & $4.8 \pm 0.7^{*}$ & $76.7 \pm 6.7^{*}$
\end{tabular}

Values represent mean \pm SEM. Abbreviation: htn, hypertension.

$* P<0.001$, comparison of long htn with control and brief htn groups.
$(0.61 \pm 0.13$ [hypertensive] vs. $0.14 \pm 0.02 \mathrm{mmHg} / \mathrm{ml}$ per $\min$ [control]; $P<0.0001$ ). Table IV shows arterial blood gas values for both groups at baseline (fetal), and 20 and $120 \mathrm{~min}$ after delivery. As shown, arterial $\mathrm{PO}_{2}$ at 120 min was significantly decreased in the hypertensive animals. Although differences in arterial $\mathrm{PO}_{2}$ between control and hypertensive animals were not statistically significant at $\mathbf{2 0} \mathrm{min}$, this may reflect the small number of animals in each group. At 120 min after cesarean-section delivery, the hypertensive animals required

Table III. Comparisons of Ventricular Weights between Control and Hypertensive Fetal Animals ( $n=12$ in Each Group)

\begin{tabular}{lccc}
\hline \multicolumn{1}{c}{ Parameter } & Control & Hypertensive & $P$ value* \\
\hline Fetal weight $(k g)$ & $3.59 \pm 0.14$ & $3.38 \pm 0.10$ & NS \\
Gestational age $(d)$ & $139 \pm 1$ & $136 \pm 1$ & NS \\
Right ventricle $(g)$ & $5.8 \pm 0.3$ & $7.4 \pm 0.5$ & $<0.001$ \\
Left ventricle plus septum $(g)$ & $11.8 \pm 0.5$ & $12.2 \pm 0.1$ & NS \\
RV/LV + S (ratio) & $0.49 \pm 0.01$ & $0.60 \pm 0.02$ & $<0.001$
\end{tabular}

* Paired $t$ test. 
Table IV. Comparison of Heart Rate and Arterial Blood Gas Measurements before and after Cesarean-Section Delivery of Control and Hypertensive Lambs

\begin{tabular}{lccc}
\hline Parameter & Fetal & $20 \mathrm{~min}$ & $120 \mathrm{~min}$ \\
\hline $\begin{array}{l}\text { Heart rate (bpm) } \\
\text { Control }\end{array}$ & $180 \pm 8$ & $227 \pm 8$ & $200 \pm 3$ \\
Hypertensive & $220 \pm 12$ & $185 \pm 8$ & $180 \pm 13$ \\
$\mathrm{pH}(\mathrm{U})$ & & & \\
Control & $7.36 \pm 0.01$ & $7.23 \pm 0.05$ & $7.37 \pm 0.03$ \\
Hypertensive & $7.33 \pm 0.02$ & $7.16 \pm 0.07$ & $7.33 \pm 0.06$ \\
$\mathrm{PO}_{2}($ Torr $)$ & & & \\
Control & $15 \pm 1$ & $106 \pm 44$ & $126 \pm 17^{*}$ \\
Hypertensive & $16 \pm 2$ & $57 \pm 13$ & $52 \pm 21^{*}$ \\
$\mathrm{PCO}_{2}($ Torr $)$ & & & \\
Control & $49 \pm 2$ & $58 \pm 7$ & $41 \pm 5$ \\
Hypertensive & $47 \pm 3$ & $65 \pm 12$ & $44 \pm 8$ \\
\hline
\end{tabular}

Values expressed as mean \pm SEM. Fetal blood gas tensions are from main pulmonary artery blood samples; newborn blood gas tensions are from descending aorta blood samples.

* $P<0.03$, repeated measures analysis of variance.

higher peak inspiratory pressures $\left(36 \pm 2\right.$ vs. $30 \pm 1 \mathrm{~cm} \mathrm{H}_{2} \mathrm{O}, P$ $<0.05)$ and rates $(59 \pm 7$ vs. $42 \pm 5$ breaks per minute; $P<0.01)$ than control animals. Differences in inspired oxygen concentration ( 1.00 vs. $0.66 \pm 0.17)$ and peak end-expiratory pressure $\left(5 \pm 1\right.$ vs. $4 \pm 1 \mathrm{~cm} \mathrm{H}_{2} \mathrm{O}$ ) were not significant between groups.
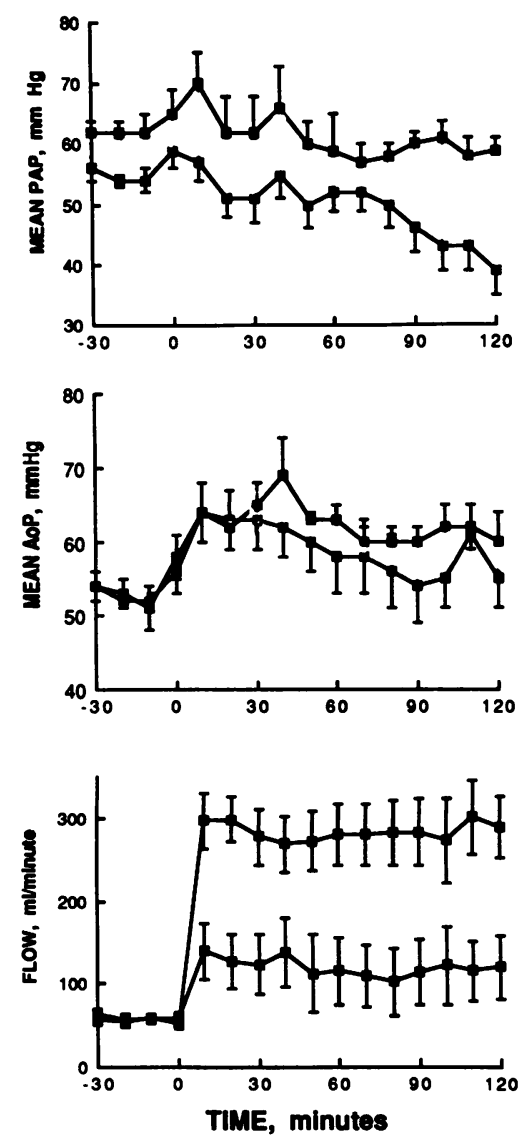

Figure 5. The hemodynamic response before and for $2 \mathrm{~h}$ after cesarean-section delivery are shown for control animals () and study animals (ם). In contrast to the control group, mean pulmonary artery (top) and aorta pressures were significantly greater throughout the postdelivery period in the hypertensive animals $(P<0.0001$ and $P$ $<0.01$, respectively). Pulmonary blood flow was significantly reduced after birth in the hypertensive animals $(P$ $<0.0001$, bottom)
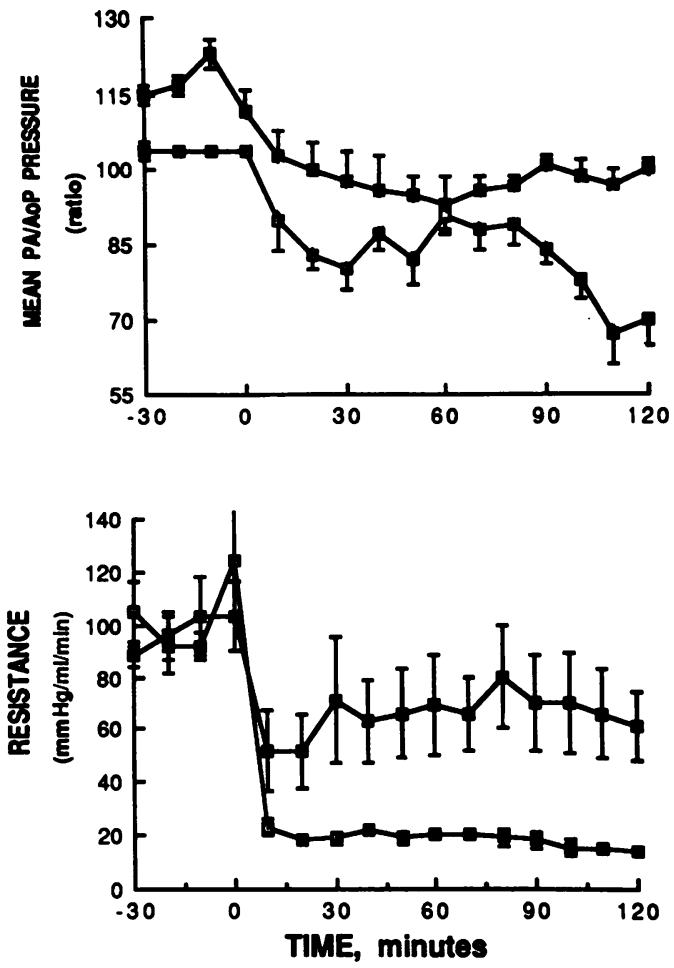

Figure 6. Changes in the ratio of mean pulmonary artery to aorta pressures (top) and total pulmonary resistance (bottom) are shown before and after cesarean-section delivery. Compared with control animals, the hypertensive group had significantly greater elevations of pulmonary to systemic pressure ratio and total pulmonary resistance after delivery $(P<0.0001$, by repeated measures ANOVA, for both comparisons).

Brief compression of the ductus arteriosus was used to assess the predominant direction of shunting across the ductus during the transition period in control and hypertensive animals. In control animals, pulmonary artery pressure and blood flow decreased during partial ductus compression, suggesting that left-to-right ductus shunting normally contributes to the rise in pulmonary blood flow shortly after birth. In contrast, the hypertensive animals had predominantly right-to-left shunting across the ductus, as demonstrated by a rise in pulmonary artery pressure and blood flow during brief ductus occlusion. In addition, each of the three hypertensive animals that had preductal arterial catheters placed after delivery had a $>20$ Torr arterial $\mathrm{Po}_{2}$ gradient between descending aorta and preductal arterial samples.

\section{Discussion}

We report that chronic partial compression of the ductus arteriosus in chronically instrumented fetal lambs causes sustained intrauterine pulmonary hypertension, leading to altered fetal pulmonary vasoreactivity, structural changes of small pulmonary arteries, and the failure of adaptation of the pulmonary circulation to postnatal conditions. These changes occurred in the absence of fetal hypoxemia and without sustained increases in pulmonary blood flow, suggesting that elevations of pulmonary artery pressure can independently lead 
to structural and functional changes. This work establishes a link between intrauterine events and the failure of postnatal adaptation of the pulmonary circulation at birth, and demonstrates histologic features and pathophysiologic abnormalities characteristic of human persistent pulmonary hypertension of the newborn $(6,7,10,14,15)$. Although previous studies have suggested that fetal events may alter either vascular reactivity $(31,32)$ or structure $(11-13,16,17)$, more recent studies have failed to confirm these observations (18-20). This report confirms the suggestions of the early studies that intrauterine stimuli can significantly alter the pulmonary vascular reactivity and structure, and is the first report to demonstrate the failure of adaptation of the pulmonary circulation at birth after chronic pulmonary hypertension.

Early clinical and pathologic observations of newborns dying with persistent pulmonary hypertension of the newborn demonstrated the striking proliferative pulmonary vascular lesions that characterize this syndrome (9), which was later quantified by rigorous morphometric analysis $(10,14,15)$. Based on these studies, it was hypothesized that chronic intrauterine stimuli, such as fetal hypoxia or hypertension, contribute to the pathogenesis of persistent pulmonary hypertension of the newborn. Goldberg et al. found smooth muscle thickening of neonatal pulmonary vessels after chronic maternal exposure to hypoxia, but their fixation method did not include vascular perfusion with fixative (11). Levin and co-workers demonstrated that elevation of fetal pulmonary artery pressure secondary to systemic hypertension or partial ductus compression, causes marked wall thickening of fifth-generation pulmonary arteries in fetal lambs (16). Similarly, partial ductus closure secondary to cyclooxygenase inhibition (15) or ligation $(16,17,33)$ leads to similar structural lesions of small pulmonary arteries. However, recent attempts to induce these proliferative pulmonary vascular lesions in newborn rats and guinea pigs after maternal exposure to hypoxia, or chronic maternal administration of indomethacin to guinea pigs, failed to result in similar lesions $(18,19,20)$. Although species differences may potentially explain some differences between studies, these latter studies lacked intrauterine measurements to confirm successful closure of the ductus, or the development of significant pulmonary hypertension. Other studies have reported changes in pulmonary vascular reactivity after chronic hypoxia, but lack histologic demonstration of structural changes or did not examine events during birth itself $(31,32)$.

By using an exteriorized inflatable vascular occluder around the ductus arteriosus, we were able to control the level of intrauterine pulmonary hypertension, thereby avoiding the development of hypoxemia which develops over time with severe partial ductus closure. In addition, as determined from serial fetal pulmonary blood flow measurements, we report that hypertension, in the absence of high flow or hypoxia, is a significant stimulus leading to the striking alterations of pulmonary vascular structure and reactivity in this model. This study extends our recent observation that although the acute hemodynamic response to partial compression of the ductus arteriosus is associated with an initial rise in pulmonary blood flow, a steady decline in flow towards baseline values occurs by two hours, despite continued hypertension (25).

Based on our morphometric analysis of small pulmonary arteries, it appears that intrauterine pulmonary hypertension over $8 \mathrm{~d}$ leads to significant structural changes. The duration and severity of pulmonary hypertension required to alter pulmonary vasoreactivity is not completely clear from these data. Based on previous observations that acute hypertension secondary to partial ductus compression blunts the pulmonary vasodilator response to small increases in fetal $\mathrm{PO}_{2}(25)$, it appears that fetal pulmonary vasoreactivity can be altered after more brief exposures to pulmonary hypertension. Whether more brief hypertension, without striking histologic changes, can lead to the maladaptation form of persistent pulmonary hypertension of the newborn is not known (8). Further studies comparing the hemodynamic response to cesarean-section delivery after more brief exposure to pulmonary hypertension may clarify whether changes in reactivity predate the appearance of striking proliferative changes of small pulmonary arteries.

In addition, we observed significant right ventricular hypertrophy after chronic ductus compression, as expressed an increase in the ratio of the weight of the right ventricle to left ventricle plus septum. Although right ventricular hypertrophy is traditionally assessed in hearts from adult animals in this manner (28), the ratio may actually underestimate the degree of hypertrophy in the lamb fetus, in which the right ventricular output represents nearly two-thirds of the combined ventricular output (4).

Despite early and aggressive treatment with high concentrations of inspired oxygen and mechanical ventilation, the transition of the pulmonary circulation remained abnormal after chronic ductus compression. Although barotrauma and hyperoxia could potentially aggravate lung injury, contributing adversely to the hemodynamic responses at birth, these stimuli alone cannot account for the differences between the control and hypertensive animals. Similarly, although this study provides experimental confirmation of the hypothesis that intrauterine events contribute to the pathogenesis of persistent pulmonary hypertension, the potential role of postnatal injury in exacerbating or amplifying pulmonary vascular injury has not been addressed. Recent studies suggest that mechanical ventilation or exposure to hyperoxia may injure the pulmonary microvasculature of newborn animals (34). Sustained pulmonary hypertension secondary to abnormal vasoreactivity could cause further pulmonary vascular injury during the postnatal period, leading to more marked structural changes with time. Although less ventilator support was needed to achieve better arterial blood gas values than in hypertensive animals, the control group required higher pressures and rates than expected. By histologic assessment, there was no evidence of barotrauma, pulmonary edema, retained lung liquid, or less structural maturity in the control group. Whether this reflects the effects of fetal surgery on lung mechanics, growth, or pulmonary vascular reactivity is not known, but demonstrates the importance of appropriate controls for studies of the transitional pulmonary circulation.

Although the narrowed lumina, medial hypertrophy, and increased perivascular adventitia most likely contribute to the sustained elevations of pulmonary artery pressure and resistance, the potential role of vasoconstricting stimuli has not been studied. During the normal transition the initial rise in blood flow and decline in pulmonary artery pressure is associated with recruitment and vasodilation within the first $24 \mathrm{~h}$ $(13,35)$. A more gradual decline in pulmonary vascular resis- 
tance is associated with vessel growth and decreased muscularity during the weeks after birth. Histologically, a reduction in cell-cell overlap, and thinning of the endothelium has been described (35). Hypertensive vessels may have undergone a structural reorganization, leading to the inability to achieve or sustain vasodilation at birth $(13,35,36-38)$.

In summary, we found that chronic intrauterine pulmonary hypertension secondary to partial compression of the ductus arteriosus leads to abnormal fetal and neonatal pulmonary vascular reactivity, structural remodeling of small pulmonary arteries, and the failure of the pulmonary circulation to adapt to postnatal conditions. This animal model has striking parallels with the clinical characteristics of persistent pulmonary hypertension of the newborn, such as $(a)$ sustained pulmonary hypertension in the early hours after birth; $(b)$ rightto-left shunting contributing to hypoxemia; $(c)$ a lack of responsiveness to $100 \% \mathrm{O}_{2}$ and mechanical ventilation; and $(d)$ histologic changes, suggesting decreased cross-sectional surface area due to narrowed lumina and thickened walls of small pulmonary arteries. We speculate that chronic partial compression of the ductus arteriosus in the fetal lamb will provide an adequate animal model for developing greater insight into the pathogenesis and pathophysiology of persistent pulmonary hypertension of the newborn.

\section{Acknowledgments}

The authors are grateful for the support and encouragement of Dr. Giacomo Meschia and Dr. Frederick C. Battaglia and technical help from Vickie Hillbrand, Lee Hatton, Y-Da Fan, and Ginger Johnson.

This work was supported in part by grants from National Institutes of Health (NIH) Studies in Prematurity (HD-00781 and HD-01869), the Colorado Heart Association, and NIH Clinical-Investigator Award (K08-HL01932), and FIRST award (1R29-HL41012-01).

\section{References}

1. Cassin, S., G. W. Dawes, J. C. Mott, B. B. Ross, and L. B. Strang. 1964. The vascular resistance of fetal and newly-ventilated lung of the lamb. J. Physiol. (Lond.). 171:61-79.

2. Dawes, G. S., J. S. Mott, J. C. Widdicombe, and D. G. Wyatt. 1953. Changes in the lungs of the newborn lamb. J. Physiol. (Lond.). 121:141-162.

3. Padbury, J., Y. Agata, J. Ludlow, M. Ikegami, B. Baylen, and J. Humme. 1987. Effect of fetal adrenalectomy on catecholamine release and physiologic adaptation at birth in the sheep. J. Clin. Invest. 80:1096-1103.

4. Teitel, D. F., H. S. Iwamoto, and A. M. Rudolph. 1987. Effects of birth-related events on central blood flow patterns. Pediatr. Res. 22:557-566.

5. Leffler, C. W., T. L. Tyler, and S. Cassin. 1978. Effect of indomethacin on pulmonary vascular response to ventilation of fetal goats. Am. J. Physiol. 234:H346-351.

6. Gersony, W. M., G. V. Duc, and J. C. Sinclair. 1969. "PFC" syndrome (persistence of the fetal pulmonary circulation). Circulation. 40(Suppl. III):87. (Abstr.)

7. Levin, D. L., M. A. Heymann, J. A. Kitterman, G. A. Gregory, R. H. Phibbs, and A. M. Rudolph. 1976. Persistent pulmonary hypertension of the newborn. J. Pediatr. 89:626-630.

8. Rudolph, A. M. 1980 . High pulmonary vascular resistance after birth: pathophysiologic considerations and etiologic classification. Clin. Pediatr. 19:585-590.
9. Siassi, B., S. J. Goldberg, G. C. Emmanouillides, S. M. Higashino, and E. Lewis. 1971. Persistent pulmonary vascular obstruction in newborn infants. J. Pediatr. 78:610-615.

10. Murphy, J. D., M. Rabinovitch, J. D. Goldstein, and L. M. Reid. 1981. The structural basis of persistent pulmonary hypertension of the newborn infant. J. Pediatr. 98:962-967.

11. Goldberg, S. J., R. A. Levy, B. Siassi, and J. Betten. 1971. Effects of maternal hypoxia and hyperoxia upon the neonatal pulmonary circulation. Pediatrics. 48:528-533.

12. Harker, L. C., S. E. Kirkpatrick, W. F. Friedman, and C. M. Block. 1981. Effects of indomethacin on fetal rat lungs: a possible cause of persistent fetal circulation. Pediatr. Res. 15:147-151.

13. Haworth, S. G. 1988. Pulmonary vascular remodeling in neonatal pulmonary hypertension: state of the art. Chest. 93:133S-138S.

14. Haworth, S., and L. Reid. 1976. Persistent fetal circulation: Newly recognized structural features. J. Pediatr. 88:614-620.

15. Levin, D. L., D. E. Fixler, F. C. Morris, and J. Tyson. 1978. Morphologic analysis of the pulmonary vascular bed in infants exposed in utero to prostaglandin synthetase inhibitors. J. Pediatr. 92:478-483.

16. Levin, D. L., A. I. Hyman, M. A. Heymann, and A. M. Rudolph. 1978. Fetal hypertension and the development of increased pulmonary vascular smooth muscle: a possible mechanism for persistent pulmonary hypertension of the newborn infant. J. Pediatr. 92:265-269.

17. Ruiz, V., G. J. Piasecki, K. Balogh, B. J. Polansky, and B. T. Jackson. 1972. An experimental model for fetal pulmonary hypertension: a preliminary report. Am. J. Surg. 123:468-471.

18. Demello, D. E., J. D. Murphy, M. J. Aronovitz, P. Davies, and L. M. Reid. 1987. Effects of indomethacin in utero on the pulmonary vasculature of the newborn guinea pig. Pediatr. Res. 22:693-697.

19. Geggel, R. L., M. J. Aronovitz, and L. M. Reid. 1987. Effects of chronic in utero hypoxemia on rat neonatal pulmonary arterial structure. J. Pediatr. 108:756-759.

20. Murphy, J. D., M. J. Aronovitz, and L. M. Reid. 1986. Effects of chronic in utero hypoxia on the pulmonary vasculature of the newborn guinea pig. Pediatr. Res. 20:292-295.

21. Abman, S. H., F. J. Accurso, R. M. Ward, and R. B. Wilkening. 1986. Adaptation of fetal pulmonary blood flow to local infusion of tolazoline. Pediatr. Res. 20:1131-1135.

22. Lewis, A. B., M. A. Heymann, and A. M. Rudolph. 1976. Gestational changes in pulmonary vascular responses in fetal lambs in utero. Circ. Res. 39:536-541.

23. Anderson, D. F., J. M. Bissonette, J. J. Faber, and K. L. Thornburg. 1981. Central shunt flows and pressures in the mature fetal lamb. Am. J. Physiol. 241:H60-66.

24. Lock, J. E., F. Hamilton, H. Luide, F. Coceani, and P. M. Olley. 1980. Direct pulmonary vascular responses in the conscious newborn lamb. J. Appl. Physiol. 48:188-196.

25. Abman, S. H., and F. J. Accurso. 1989. Acute effects of partial compression of the ductus arteriosus on pulmonary blood flow and vasoreactivity in fetal lambs. Am. J. Physiol. In press.

26. Abman, S. H., F. J. Accurso, R. B. Wilkening, and G. Meschia. 1987. Persistent pulmonary hypoperfusion after acute hypoxia. $\mathrm{Am}$. J. Physiol. 253:H941-948.

27. Accurso, F. J., B. Alpert, R. B. Wilkening, R. G. Peterson, and G. Meschia. 1986. Time-dependent response of fetal pulmonary blood flow to an increase in fetal oxygen tension. Respir. Physiol. 63:43-52.

28. Fulton, R. M., E. C. Hutchinson, and A. M. Jones. 1952. Ventricular weight in cardiac hypertrophy. Br. Heart J. 14:413-420.

29. SAS User's Guide. 1982. Statistics. SAS Institute, Cary, NC.

30. Sheldon, R. E., L. L. H. Peeters, M. D. Jones, E. L. Makowski, and G. Meschia. 1979. Redistribution of cardiac output and oxygen delivery in the hypoxemic fetal lamb. Am. J. Obstet. Gynecol. 135:1071-1078.

31. Drummond, W. H., and J. M. Bissonette. 1978. Persistent 
pulmonary hypertension in the neonate: development of an animal model. Am. J. Obstet. Gynecol. 131:761-763.

32. Soifer, S. J., D. Kaslow, C. Roman, and M. A. Heymann. 1987. Umbilical cord compression produces pulmonary hypertension in newborn lambs: a model to study the pathophysiology of persistent pulmonary hypertension of the newborn. J. Dev. Physiol. 9:239-252.

33. Morin, F. C., and L. Wilde. 1988. Persistent fetal circulation (PFC) in the newborn lamb: physiologic and anatomic replication of human PFC. Pediatr. Res. 23:436a. (Abstr.)

34. Dreyfuss, D., G. Basset, P. Soler, and G. Saumon. 1985. Intermittent positive pressure hyperventilation with high inflation pressures produces pulmonary microvascular injury in rats. Am. Rev. Respir. Dis. 132:880-884.
35. Hall, S. M., and S. G. Haworth. 1986. Normal adaptation of pulmonary artery intima to extrauterine life in the pig: ultrastructural studies. J. Pathol. 149:55-66.

36. Mecham, R. P., L. A. Whitehouse, D. S. Wrenn, W. C. Parks, G. L. Griffin, R. W. Senior, E. C. Crouch, K. R. Stenmark, and N. F. Voelkel. 1987. Smooth muscle mediated connective tissue remodeling in pulmonary hypertension. Science (Wash. DC). 237:423-426.

37. Allen, K., and S. Haworth. 1986. Impaired adaptation of intrapulmonary arteries to extrauterine life in newborn pigs exposed to hypoxia: an ultrastructural study. J. Pathol. 150:205-212.

38. Meyrick, B., and L. Reid. 1982. Normal postnatal development of the media in the rat hilar pulmonary artery and its remodeling by chronic hypoxia. Lab. Invest. 46:505-516. 